\title{
Becoming Digital: On the Importance of E-material in Transforming Preconceptions about Academic Research
}

\author{
Mariem Khmiri \\ The Higher Institute of Applied Studies in Humanities of Sbeitla, University of Kairouan, Tunisia.
}

\begin{abstract}
How to cite this paper: Mariem Khmiri. (2021) Becoming Digital: On the Importance of E-material in Transforming Preconceptions about Academic Research. Journal of Humanities, Arts and Social Science, 5(1), 6-15.

DOI: 10.26855/jhass.2021.01.002
\end{abstract}

Received: November 23, 2020

Accepted: December 29, 2020

Published: January 13, 2021

*Corresponding author: Mariem Khmiri, The Higher Institute of Applied Studies in Humanities of Sbeitla, University of Kairouan, Tunisia.

Email: merriemkhmiri@hotmail.com

\begin{abstract}
The post-print era was peculiar for a dramatic shift in the study of the humanities. Concomitant with this insurgency called digitization was the inscription of ideology on the minds of its subjects which succeeded in disconcerting many of those who spoke confidently for a digital revolution in the academic context. The debate whether digitized knowledge should be scrutinized in a competitive or a cooperative light has brought with it a certain indeterminacy of definition which allows it to figure in a varied span of concerns: self and other, past and present, sign and concept, etc. My argument is that the category of the digital assumes the polysemy it does in our modern world because, in speaking of the humanities, it speaks also of power relations which are at the heart of the struggle for cultural hegemony. Ultimately, in its cooperative or competitive underpinnings, the subjectivity of the user is the epistemic source most actively engaged in producing and extracting meaning in a digital context.
\end{abstract}

\section{Keywords}

Historicity, Ideology, Power Relations, Cooperative, Competitive, Past, Present, Sign, Concept, Preconception

\section{Literature Review}

In an article entitled “Long-Term Digital Preservation: A Digital Humanities Topic?”, Henry M. Gladney speaks of a likely outcome of the digitization of the humanities as the failure to implement "institutional change": a failure which stems (according to him) from a cultural incompatibility more than a lack of technical skill. Gladney's point is that social intelligence is (and should be gauged) an ingredient of capital importance next to the legibility of technology as a science. It seems that Gladney suspects digitization to be a disadvantageous act which transforms literary data into a fixity: it might therefore turn into an impediment to social intelligence and consequently to what he calls "institutional change". Gladney, however, seems also to miss the more pragmatic question around "institutional change" in the extent to which the archiving of the humanities actually converts data into a "fixity" (the term is by Gladney) or not: that is the extent to which archiving (as a technique) ever allows a bridge to be thrown between digitized humanities (on the one hand) and literary interpretation (on the other).

I opine that Gladney fails to recognize the very flexible nature of digital humanities whose archiving never isolates them from the workings of interpretation. I am also suggesting that this flexibility is what brings forth another approach to digital humanities as an ideological artifact: hence the prospect of "institutional change". I see the root of the problem in how Gladney maintains that archives are one thing and "new" data are another. I will suggest that 
digitized data (albeit archived) is far from being a "fixity". Subjecting data to a wide range of transformations by the mere expedient of having them digitized is what also helps me signal an issue with Gladney in how he implicitly reduces digitization to a state of crisis. As a result, and contrary to Gladney's pessimism, I am also acknowledging the progress in time of Digital Humanities (i.e., the historicity of Digitized Humanities) as the very precondition of "institutional change”. Finally, ideology will be revealed as one of the necessary manifestations of this institutional change and is thus another response to Gladney's idea of digital archives as a fixity.

In their article "Learning to Read Data: Bringing out the Humanistic in the Digital Humanities”, Ryan Heuser and Long Le-Khac postulate that the hunt for word frequency in a digital text is a full-fledged grantor of meaning. Personally, however, I find in the insidious reductionism behind their (Heuser and Le-Khac's) obsession with counting words an urgent invitation to recognize ideology (instead) as the most engaging meaning-maker. I see in the subordination to a system of signs (in the name of a digital revolution) a formal articulation of the mystified guise of the ideological apparatus underlying the digitization of the humanities. Todd Presner responds negatively to the quantitative method used by Heuser and Le-Khac. Actually, Presner's evocation of Theodor Nelson's notion of hypertext is a key to a qualitative, therefore more inclusive-grasp of the digital humanities.

I hypothesize that the illusion of complete autonomy while deploying a digital medium of any sort should be soon discarded for the more compelling reality about ideology as the bottom-line of any digital preoccupation. What this reality entails, nevertheless, is the ongoing anxiety among critics whether Digital Humanities is a cooperative or a competitive form of social relations.

\section{Research objectives}

The present paper attempts to resist the cliché that the digitization of the humanities has brought forward some breakthrough in the twentieth century. This resistance on my part is due to the historical fact that the digital age is also purely a property of the industrial revolution. Therefore, I am rather concerned with the digital humanities as a destabilizing cultural controversy which has thrown up a trend of talking about literature often at odds with its cultural antecedents. My argument is that recognizing the ongoing debate between past and present candidly heightens the visibility of the curious interactivity of the digital work with its past.

\section{Research questions}

The current study is an attempt to answer the following questions:

- $\quad$ To what extent is the discipline of Digital Humanities a cultural problem?

- $\quad$ How can the historicity of Digital Humanities be approached as a modern phenomenon?

- $\quad$ How do the Digital Humanities challenge the duality of sign/concept?

- How can the Digital Humanities be a manifestation of ideological practices? Can anything be done to stave off this risk?

- Has the debate whether the Digital Humanities are a cooperative or a competitive interface finally come to a close? Or has the compulsion of its scholarly environment finally managed to shape into a harmonious unity the curiously unstatic content of the Digital Humanities?

\section{Part One: Digital Humanities as competitive}

\section{Gladney's argument about Digital Humanities as a cultural crisis}

In an article entitled “Long-Term Digital Preservation: A Digital Humanities Topic?”, Henry M. Gladney has delineated the reception of digitization in the field of the humanities essentially as a cultural shock: what he euphemistically referred to the slow pace of "institutional change" in an excerpt he quoted from Wikipedia (Gladney, 2012, p. 201). He then clarifies his point by relating this sudden unease produced by digitization to a misunderstanding of the digital alphabet: therefore essentially to a cultural lag:

Intellectually it has to do with the poorly understood nature of non-verbal knowledge-bearing objects. Curatorially it raises the problem of how such knowledge-bearing objects are to be preserved for the long term. Culturally it runs afoul of the low status given to works of popular culture -multimedia, documentaries, interactive games, and [so on]-which tend to be dismissed as entertainment.

(Gladney, 2012, p. 201)

It is clear from this opposition set by Gladney between digital culture (on the one hand) and the scant level of understanding among many of its contemporaries (on the other) that he is trying to give a broad, interdisciplinary 
definition of digitization from a mere thing of the brain to something of a cultural dimension: that is by giving attention to a socio-cultural crisis next to that of human intelligence.

Let us begin by admitting that Gladney's definition of digitization is an implicit credit to social intelligence next to the mechanics and electronics of technology. I insist on the nominal group "social intelligence" (or interactive intelligence) as what gives shape, therefore more efficiency - to technology. Gladney is inclined to humanize the discipline of information technology. According to Gladney, human interaction is what bestows on technology the quality of being a "science" in the full sense of the term: which is actually true:

Within information science, attention has been given in recent years to human computer interaction, groupware, the semantic web, value sensitive design, iterative design processes and to the ways people generate, use and find information.

(Gladney, 2012, p. 203)

This is how Gladney seeks to display his primary concern about the social streak of information science as something vying for prominence next to the computer as a machine (Gladney, 2012, p. 203). Indeed, the term information goes some distance in answering the importance of social life alongside technology as a science. It is in this light that I make sense of digital humanities as something competitive: I speak of an extant competition between the technological and the interactive energy within the business of digital humanities. Each of the two energies is competing with the other to gain preeminence over human life.

Among the questions addressed by M. Gladney is the following: "What criteria must be satisfied for world-wide digital preservation practice to be judged socially satisfactory?” (Gladney, 2012, p. 206). In fact, by addressing the idea of being "socially satisfactory" Gladney is already pointing to the interactive aspect of digital material also as a property of social life. Digital material is implemented in social life and ipso facto in the historical changes affecting human society: from an agrarian to an industrialized society until the advent of the digital age.

Another level of rivalry is between the long-preserved corpuses of literature written on paper (on the one hand) and the compression of data thanks to technology (on the other):

Today, complex data -its form, manipulation, and interpretation- are as important to humanities study as more traditional research materials: digitized historical records [and] multimedia collections are increasing in number due to the affordability of mass data storage devices, extensive networking capabilities, and sophisticated [software] improving interactive access to and analysis of these data.

(Gladney, 2012, p. 204)

Gladney reacts to the debate about digital humanities by calling for adaptation as a solution to technological novelties (Gladney, 2012, p. 204). I may add that what he suggests as "adaptation" is much akin to an acculturation process. It is about the adaptation to technology as a new cultural trend: hence another ongoing competition can be brought in the limelight: namely between tradition (on the one hand) and modernity (on the other).

\section{Gladney's misreading of the historicity of digital humanities}

As a form of social behavior, digital collection and storage bring the objective side by side with the subjective aspects of information science. However, this encounter means also that technology does bear out the traces of its historical time. In other words, digital humanities are in a ceaseless process of change. This is the remark Gladney fails to make about the excerpt he took from Duranti:

The ease with which [dynamic documents] can be manipulated has given ... a new reason for keeping them: "repurposing" ... We have to consider the possibility of substituting the characteristics of completeness, stability and fixity with the capacity of the [repositories] to trace and preserve each change the record has undergone. (Duranti, 2004 qtd in Gladney, 2012, pp. 209-210)

The following exclamation:

We disagree! Neither our careful definition of "authenticity” nor any other work suggests that "dynamic documents” (representations of artistic and other performances) present a new or difficult preservation problem. (Gladney, 2012, p. 210)

—offers insight about Gladney's misunderstanding of the inherently mobile nature of digital humanities. I say mobile because (as a science) digital humanities easily yields itself to the workings of time. More than that, a "preservation problem" is always concomitant with digitizing data. The reason is that digitized data is to be updated or 
else it risks oblivion. Again, Gladney's complacent remark in the above exclamation betrays his misreading of the historicity of digital material as (what he mistakes for) a potential threat to authenticity.

Mobility is an ongoing process of change within the timeline to which Digital Humanities is highly permeable. Gladney's insouciance of the effect of time on digital humanities (and of the variegated range of interpretations they invite each time they are updated) is at the very heart of his problem of logic:

By scholarly multimedia we specifically mean critical scholarly works -interpretive and argumentative, as opposed to creative or archival -that are produced, and perhaps performed in multimedia form. (Gladney, 2012, p. 205)

Contrary to Henry M. Gladney's monolithic idea about digital humanities (and in an article he wrote as a contributor to the electronic blog of the New York Times: Opinionator), Stanley Fish (2012) captured the differences in the interpretation of "data" across the traditional and the digital practices in a very conciliatory string of words:

Digital Humanities: an umbrella term for new and fast-moving developments across a range of topics [...] The expansion of digital archives, the refining of search engines, the production of scholarly editions, $[\ldots]$ the transformation of scholarly publishing $[\ldots]$ the redefinition of what it means to be a text.

Similarly to Stanley Fish who reveals the importance of Digital Humanities as a flexible and self-regenerative unit of meaning (by virtue of its openness to "restructuring", "transformation" and "re-conception"), Sabharwal has also mentioned the importance of placing data in its historical context:

Treating data as interpretable text is also close to the mission of archives active in curation and outreach. Exhibitions, lecture series, presentations, and other educational events at archives place data into a historical context. (Sabharwal, 2015, p. 21)

Digital Humanities is the kind of discipline which compels the examination of its purpose in full light of its historical time. In his article "Cultural Diversity and the Digital Humanities" (2018), Simon Mahony looks at digital humanities as a historical truth in its own right. He takes it as the kind of venture which conjoins the technological side by side with the human. As such, and in the Mahony optic, Digital Humanities is ultimately a division of scholarly concern whose interest is to depart from "its historical origins in text-based scholarship" (1) towards more "openness and inclusivity" (ibid). With English as its lingua franca, the evolution of computer-mediated material in the humanities (theater, cinema or prose) has made it possible for students and teachers alike to have access to a large variety of material for their coursework thanks to the technique of archiving. The latter, thanks to the advent in technology, has marked an important leap in the field of academic research. This is also one way to have a strong feel of the humanistic turn of digitization as a user-friendly tool of academic research.

\section{Gladney's historically-decontextualized approach to Digital Humanities}

Gladney makes a statement of purpose that his essay aims to spot the "weakness" of "proposed Digital Humanities research agendas” while he also complacently argues that "long-time Digital Preservation” is a "solved challenge”:

The current article illustrates the weakness of proposed Digital Humanities research agendas by showing that Long-time Digital Preservation -the most prominently featured specified topic in recent DH articles-is a solved challenge for which all that still needs attention is software creation and deployment. (Gladney, 2012, p. 214)

In response to this absolutist and self-congratulatory claim, I need to draw attention to the ever-changing historical reality of a preserved digital material as nothing milder than a full-fledged problem. In the above quote, Gladney has fallen prey to a serious problem of logic by pretending to discuss what he views as an already-solved issue. I hope therefore to conduct a fertile line of inquiry into Gladney's self-claim by revealing that the digital preservation of data can never be brought to a close because - as a human thing - it is always subject to interpretation especially in terms of power relations. In her article "Smart Data for Digital Humanities”, Marcia Lei Zeng brings under close scrutiny the qualitative distinction of data:

“the terms 'data' and 'digital data' are not equivalent. The types of data are also not limited to quantitative data. The Reference Model for an Open Archival Information System (OAIS) defined data as a "reinterpretable representation of information in a formalized manner suitable for communication, interpretation, or processing.” (Lei Zeng, 2017, p. 8) 
In light of Lei Zeng's remark, we understand that data are always remodeled in function of the ruling thoughts of a given era. By conceiving data as a "reinterpretable representation of information", Lei Zeng is pointing to the malleability of data which — once archived — can also be retrieved and re-utilized to "represent" whichever ideology that is aimed by the authorities of a social group. It follows that the discussion of technology is not possible without a parallel discussion of how it implements power relations. In the same way that seafaring and the ship industry were behind colonial expansionism with a purview to "urbanize" and to "tame" barbaric sub-nations; the widespread of technology has for soft underbelly the formation of power relations and-more subtly — the transmission of ideological messages. Digital humanities is therefore one among the platforms where we can investigate the methods according to which ideology is planned, articulated then acted out.

In line with the argument presented by Jan Blommaert on discourse, I can add the distinction that digitization is part of the institutional (therefore material) environment which is expected to thrive alongside the "ideational or cognitive component in ideologies” (Blommaert, 2005, p. 163). As a field of education, Digital Humanities is a mirror to how the standardization of culture is worked through. This point was evoked primarily by Bourdieu who emphasizes the systematic connection between education (on the one hand) and the institutionalization of a so-called legitimate knowledge typical of the predominant culture (on the other):

Educated people owe their culture-i.e., a program of perception, thought and action— to the school.

(Bourdieu, 1971, p. 199)

This thought is obviously rooted in Foucault's theory on power and knowledge. According to Michel Foucault, what appears as the homogenous "face" of the history of a period (Foucault, 2004, p. 8) hides another facet to itself such that:

One and the same form of historicity operates upon economic structures, social institutions and customs, the inertia of mental attitudes, technological practice, political behavior, and subjects them all to the same type of transformation. (Foucault, 2004, pp. 8-9)

Foucault's historical stance is valid for Digital Humanities insofar as we consider the latter as a vehicle to present a homogenous facet of a specific historical period. Digital Humanities as a sample of "social institutions and customs" is a propitious ground for the transformation within the minds of people: that is for the flourishing of the guiding thoughts and culture of the superstructures in a given society. Foucault's mention of "technological practices" as a platform for the exercise of ideology conveys his conviction that technology can be a vector of the political, economic and social agenda of people in power.

It follows that what Foucault has delineated as the connection between an "episteme" (on the one hand) and the institutional practices which concretize it (on the other) can be actually the case for the digital humanities. Paul Gilroy has observed the same complex structure of power and knowledge within the field of digital humanities (Gilroy, 1993, p. 2). Foucault's remark about the "transformations that serve as new foundations: the rebuilding of foundations" (Foucault, 2004, p. 5) is much of an engaging statement about the necessity to view digitization within a bigger-actually historical-process of change. We make sense of the competitive edge of Digital Humanities by the mere fact that society no longer relies on the muscle or the memory of fellow humans but on the electronic memory to preserve information from oblivion: also to implement an entire system of thought and of conduct among its contemporaries.

\section{Part Two: Digital Humanities as cooperative}

\section{The historical necessity of a cooperative approach to Digital Humanities}

Following Bergson, man is destined to suffer his own alienation from this world precisely because he belongs to it: an idea Bergson shares with Camus, Nietzsche and Schopenhauer: father of nihilism. Bergson says that man is doomed to represent time with the spatial symbols we find on the dial of a clock with a desperate view to stave off the risk of alienation and of self-loss. Bergson also sanctions the relationship between the subject (man) and the object (the clock) as one of correspondence and even of endosmosis:

Now, let us withdraw for a moment the ego which thinks these so-called successive oscillations: there will never be more than a single oscillation, and indeed only one single position of the pendulum, and hence no duration. Withdraw, on the other hand, the pendulum and its oscillations; there will no longer be anything but the heterogeneous duration of the ego, without moments external to one another, 
without relation to number. (Bergson, 2001, p. 108)

The clock is a mechanical representation of something abstract: time, which yet gives assurance to man that he is not "heterogeneous" to the world where he lives. By analogy, let us withdraw the computer from a man's life only for a little while. In this case, there will be no longer anything but the "heterogeneous" individual with no access to distinct pieces of information, "without relation to number" (Bergson, 2001, p. 108): therefore with no science and consequently, with no knowledge. The alienation of the self (visualized by Bergson in case we lose track of clocktime) can be applied to the field of Digital Humanities. It ends up that either machines - the clock or the computer - is a solace to man: actually the umbilical cord connecting man to the world.

I can say that technology is a mechanism of defense invented by man against the sweeping ways of time (actually against his imminent alienation). Robert Edmond Jones' anthropological reading of man's relation to history yields the more pressing certainty about the need to always go forward with technology:

These are the days of the candid camera and the comic strip and television and reducing diets and strange new dance-steps. We have to work in the theatre of our own time with the tools of our own time. (Robert Edmond Jones, 2004, p. 19)

Robert Edmond Jones looks into the modern world in full light of its technological inventions and how they helped us refigure our artistic representations. In the course of his analysis of the theater of today, Robert Edmond Jones imagines a historically-remote scene from the Stone Ages. He depicts a group of people huddling around the fire and ready to defend themselves from wild beasts. This visualized scene is the telltale of more than just one thing.

In old times, precisely during the Stone Ages, people used to live in caves. Then, gradually, they learned to organize themselves in tribes, and better than sitting together around the fire in fear of a potential attack from a wolf or a lion, they would move from one spot of land to another in search for water supply to eke out their agriculture. In this sense, we witness the transition from the risks of hunting to the routine of settlement in search for arable lands. And with it, there was the awakening of those primitive nomadic peoples from the cannibalistic hunt-thirst to the more productive interest: agriculture. This anthropological reading of the development of human life conveys the shift from a competitive instinct of survival into the cooperative need for settlement conditioned by the search for sunshine and water supplies (Wells, 1938, pp. 56-57).

With the progress in time, there was a simultaneous development in the needs of humanity also in the tools they used to fulfill those needs. In the quote above from his book, The Dramatic Imagination, Robert Edmond Jones tells us that the tradition of theater has gone through the same process: that is hand in hand with the transition from hunting to a herding life. Within the field of drama, primitive people used to replay hunting scenes using a sword or spear: whereas modern people will dramatize some modern issues with modern techniques (like cameras, modern furniture, etc).

The mechanical revolution came out as a reinforcement of the idea that humanity needs no longer to rely on tribal or communal life but on the individual capacity for self-management. Correspondence was now to be found with the machine rather than with the muscles of fellow men and women as in the primitive ages. As we progressed on the timeline, the digitization of the humanities inaugurated a new era in the history of mankind marked off by a significant severage from the instinct of rivalry over ownership (the way it was dramatized in primitive theater, for example) into the more peaceful and humanely-informed possibilities of exchange among the users of information.

\section{Digital Humanities as an interface between orality and reality}

At this level of my analysis, I invite my readers to approach the academic responsibility toward digital material in the humanities not only in terms of man's instinctive competitiveness accentuated with the mechanical revolution but also in terms of man's cooperative inclinations which make it possible for humanity to advance as long as it also learns and teaches the worth and pleasure of sharing. Cooperativeness in the digital world is a condition not an exception.

Coming now to theater and the tradition of storytelling (and building on the argument on the development of dramaturgy by Edward Jones), I can say that digitization has had its impact on the very quality of performance given to its recipients. A landmark in the history of drama was the advent of photography. This was in the year 1840 when a man named David Hill discovered how to make a thing he called a photograph. The sense of cooperation came from this voyeuristic drive in man: which makes him perpetually on the lookout for details about other people's lives. In drama, it is the desire to see actors speak and move onstage the way they do in daily life.

So, similarly as it happened that all art became influenced by the invention of the photograph, people became also 
fascinated by the lure of capturing the immediacy of the moment. It is the lure of pictures in how they communicate things as they are and (to a large extent) spare people the trouble of telling stories and the risk of having those stories altered while handed over from one generation to the other within the oral tradition. What humanity, in my opinion, has developed is this sharp fear of losing some particles of the text (any text) and it seems that the digitization of the world of art—starting with photography—has given them this assurance. I speak of the assurance of fixity: which is in keeping with Bergson's idea about the alienation of the self due to his keen time-consciousness. It follows that technology (whichever form it takes: a clock, a photograph or a computer) came out to alleviate this fear of self-loss: of being swept off by the inexorable flux of time.

What digitization offered the world is also a fresh sense of reality. People are no longer in pursuit of reported things but rather crave for immediacy. All these ambitions were at once stirred and realized by the photograph, then gradually by the video tape until the computer came into history.

\section{From sign to concept: the self-undoing hunt for word frequency by Heuser and Le-Khac}

In their article: “Learning to Read Data: Bringing out the Humanistic in the Digital Humanities”, Ryan Heuser and Long Le-Khac examine the cooperative aspect of digitization in how it renders literary interpretation easier and therefore more efficient. Their focal point is the traceability of information through statistics: word frequency being a very prominent example. According to them, an intimate correlation is created within computer science between the countability of signs (as a property of technology, on the one hand) and the filament of concepts (as a property of the humanities, on the other):

A signal is the data from the feature actually being measured computationally. A concept, however, is the phenomenon we take a signal to stand for. In the digital humanities, the interest and impact of our arguments are based on concepts, but computers can only measure signals, which are always smaller than concepts.

(Heuser \& Le-Khac, 2011, p. 81)

This parallel set by Ryan Heuser and Long Le-Khac between the quantitative and the qualitative aspects of Digital Humanities enables the two researchers to come to grips with the cooperative quality of technology once applied to the field of the humanities. This is the very merit which (according to them) bridges the gap between two initially distant rives: namely signs and concepts.

However, Ryan Heuser and Long Le-Khac point with their index to the task of bridging this gap as no less than a "problem". In so doing, they are skeptical about the often inefficient way wherewith technology sometimes kisses the field of the humanities. Consequently, they hypothesize that the reality of Digital Humanities is grounded in the sign system of the computational world and in our ceaseless propensity to test whether this sign system fits (or not) within the mould of our established concepts:

The essential problem of quantitative evidence, then, is in deciding how to bridge the perpetual distance between the signals we have and the concepts we want them to represent [...] a familiar concept is applied too hastily to the data, thus flattening the data's nuances and complexities. A troubling corollary to this is a tendency to throw away data that does not fit our established concepts. (Heuser \& Le-Khac, 2011, p. 81)

Ryan Heuser and Long Le-Khac soon discard this doubt in favor of the more rational intuition that a "new knowledge" is always to be born from this fecund encounter between the humanities and the digital world:

If we required all data to make sense — that is, fit our established concepts quantitative methods would never produce new knowledge. (Heuser \& Le-Khac, 2011, p. 81)

The cooperative merit they attach to the hypertext immediately calls to mind another aesthete: Johanna Ducker who-in an interview she conducted with Roberto Simanowski about digitial humanities versus traditional literature-insists on pursuing the truth about digitization always as a medium for a message. She abstains from all stigmatization of the epithet "digital" and maintains an engaging stance about the digital world as what always generates a new meaning to a work of art in any field:

Just that the stigma of the "digital” went away so we can just think about the works as art-are they interesting, engaging, successful. We don’t talk about oil paint art or instrumental art, so why emphasize a medium or technique?

(Roberto Simanowski, 2016, p. 54) 
Johanna Drucker is of the camp which is heedless whether a sculpture is made of marble or whether its three dimensions are digitized. In this light, her cooperative consciousness drives her argument towards a digitization as a new epistemic source. As to Heuser and Le-khac, we cannot deny the fact that what they suggested as the "new knowledge" generated by the numerical method is very limited in kind if not downright unpromising. By calling us to count signs to draw a limited number of interpretations, Ryan Heuser and Long Le-Khac paradoxically scupper their initial promise to produce "new knowledge":

Scaling up the number of word frequencies being tracked, for instance, helps limit the number of possible interpretations. Imagine finding not several but hundreds of words sharing one trend. As the number of words undergoing the same change increases, the number of causes that could plausibly have affected all of those distinct words decreases. Maximizing a signal's conceptual coherence helps in making potential causes identifiable. (Heuser \& Le-Khac, 2011, p. 82)

The remark made by Heuser and Le-Khac (that limiting the number of interpretations of a digitized piece of literature by counting words is advantageous) reveals their own view of the digitization of the humanities as essentially too simplistic:

Thus, the clearer the links between words, the easier it is to identify the probable cause by examining those links. (Ibid)

Word frequency is not the right thermometer to interpret the range of meanings sprung from a piece of literature. The migration from signs to concepts (or from numbers to meanings as in the logic of Heuser and Le-Khac) is hardly a viable generator of knowledge. It ends up that there is no such a thing as a "readily interpretable form" (Heuser \& Le-Khac, 2011, p. 84). The digitization of a text (of any genre) should not be taken as a pretext to systematize knowledge according to a ready-made plan or preconception. Contrary to that, knowledge about a text (digital or not) is an endless process whose sole arbiter is the reader.

Digitization brings about the assurance of survival on the timeline for any piece of literature. It thus finds meaning to itself as a method of safeguarding information with a view to preserve it for upcoming (actually never-ending) interpretative endeavors.

\section{Todd Presner: beyond the forçage of preconceptions upon Digital Humanities}

In his article, "Comparative Literature in the Age of Digital Humanities: On Possible Futures for a Discipline”, Todd Presner brings the question of digital humanities under positive light. He makes a reasonable point by recognizing the synergistic aspects of the digital world as a platform to the exercise of ideology then also as a propitious ground for the democratization of knowledge:

[E]very technology has a dialectical underbelly, facilitating a potential democratization of information and exchange, on the one hand, and the ability to exercise exclusionary control and violence on the other. (Presner, 2011, p. 194)

Todd Presner is biased towards a comprehensive view of digitization based on the positive impact it leaves on its contemporaries: precisely in how it allows them to exchange data and most of all, to develop any input into a more sophisticated stock of knowledge. It is clear therefore that in Presner's view, the question of Digital Humanities hardly falls outside the luminous compass of cultural interactivity:

This is not to say that digital media is somehow more "evolved" than print media or that books are obsolete; rather it is to insist on the multiplicity of media and the varied processes of mediation and remediation in the formation of cultural knowledge and the idea of the literary. I strongly believe that we -as literary scholars-need to [...] interrogate the status of knowledge, the concept of culture, and the redefinition of the social in our global information age.

(Presner, 2011, p. 199)

Pressner proceeds with his argument in favor of Digital Humanities by pointing out the value of hypertext probably as a tangible proof about the cooperative character of information technology. He quotes the coinage "hypertext" used by Theodor Nelson in 1965 to refer to a fine technique which is exclusive to digital communication where individuals enjoy, in every respect, the very practical compilation of a written body of knowledge. For Nelson, a hypertext is a:

body of written or pictorial material interconnected in such a complex way that it could not conve- 
niently be presented or represented on paper [...] Such a system could grow indefinitely, gradually including more and more of the world's written knowledge. (Nelson, 2004, pp. 134-45, qtd. in Presner, 2011, 200)

Nelson presumes the possibility of growth, of development in any form of cognition offered by the digitized data. An optimistic consensus is therefore brought into being within the argument shared by Nelson and Presner in favor of the ever-flowing movement of Digital Humanities. Their consensus is nourished by the promise that the digitization of data (with all its apparent newness and distance from written data) is actually following a very humanistic trajectory. Digital Humanities is therefore an organism in its own right, positioned in the academia in a way quite above the reproach of a rigid scientific knowledge of an objective reality.

It is possible to set the argument of Todd Presner against that of Ryan Heuser and Long Le-Khac in more than one respect. While the latter conceive of an immediate one-to-one correspondence between digital signals (like word frequency) and concept-formation during (for example) the interpretation of a novel; Presner is keen on adopting a more flexible view towards the scientific property of Digital Humanities. Presner's reference to Theodor Nelson in the course of his discussion of the hypertext as the kind of electronic medium which respects the interactivity between the user and the data at hand is a response to how Ryan Heuser and Long Le-Khac were fixated on the quantitative evidence available in the digital material and who always insist on testing preconceived ideas against emerging ones:

The essential problem of quantitative evidence, then, is in deciding how to bridge the perpetual distance between the signals we have and the concepts we want them to represent. (Ryan Heuser \& Long Le-Khac, 2011, p. 81)

The violence implied in the relative clause: "we want them to produce" (in the quote above) is a blow to the very scientific claim about the numerical method discussed by Heuser and Le-Khac. The problem with Heuser and Le-Khac is that what they view as scientific within the business of digital humanities is also totally impermeable to new possibilities of meaning. It is contrary to the narrowness of a mind which measures blooming data against preconceived (therefore fossilized) thought that Presner sets his argument with a view to accommodate the reader of digital humanities with the freedom typical of the thinking subject. Presner retains the belief in a subjective law which governs digital humanities to the extent that meaning is immediately "staged in the Digital world" (Presner, 2011, p. 203). He seems in this sense to rhyme with the point made by Ester van Laar and Alexander J. A. M van Deursen about the primacy of $21^{\text {st }}$ century learning skills over computer technology. The latter in their optic, is meant only to corroborate (not to complete) the "broader" amass of knowledge we have acquired by the children of the $21^{\text {st }}$ century:

21st-century skills are broader than digital skills [...] Besides, the 21st-century skills are not necessarily underpinned by ICT, while digital skills or literacy do provide such integration. ～(Van Laar \&

Van Deursen, 2017, p. 21)

In the same vein that Van Laar and van Deursen avoid the idea of technological determinism, Todd Presner envisions digital humanities only as a pre-condition for the establishment of strong social bonds via forums and interfaces: that is, thanks to the easy exchange and evaluation of digital data:

We no longer just browse and passively consume predigested content but are actively engaged in the production, annotation, and evaluation of digital media and software thanks to the open-source movement.” (Presner, 2011, pp. 202-203)

However, this organic liaison envisioned by Presner between the digital world (on the one hand) and the bliss of social collaboration (on the other) is quick to pale to arrant nonsense when he concludes his article on a crass note about Wikipedia as "a model for rethinking collaborative research and the dissemination of knowledge in the Humanities and at institutions of higher learning” (Presner, 2011, p. 204). Presner's denial of the fact that Wikipedia can often be amateurish and unreliable ultimately enfeebles his socially-informed sensibility about digital humanities.

\section{Conclusion}

The evolution from a herding to an industrial, then to a digital world engendered a concomitant growth in the field of the humanities. In theater, for example, modernity has brought with it the reality that people no longer wish to hear about kings and queens "hedged with divinity" (Hamlet) but are rather in need of the more immediate imprints like the effrontery they find for example, in Beckett's drama. The synergistic advent of the digital age together with-for example - the theater of the absurd has set into motion the competitive edge of modernity beyond the pang of nos- 
talgia for a so-called meaningful past. However, synonymous with digitization was also the revival of old literature. Hence the merit of digitization which finds shape in the practicality of the analytic, evaluative and communicative tools wherewith we approach digital literature: hypertext being a case in point. People should counter the tide of preconceptions in favor of the inherently cooperative truth about the Digital Humanities where self-generative knowledge is always possible.

\section{References}

Bergson, Henri. (2001). Time and Free Will: an Essay on the Immediate Data of Consciousness. Trans. F. L. Pogson. New York: Dover Publications.

Blommaert, Jan. (2005). Discourse: A Critical Introduction. Cambridge: CUP.

Bourdieu, Pierre. (1971). "Systems of education and systems of thought." Knowledge and Control: New Directions for the Sociology of Education. Ed. M. Young. London: Collier-Macmillan.

Duranti, Luciana. (2004). "The Long-term Preservation of the Dynamic and Interactive Records of the Arts, Sciences and E-Government.” Documents Numériques, 8.1: 1-14.

Fish, Stanley. (2011). “The Old Order Changeth.” The Opinionator (New York Times). December 26, 2011. http://opinionator.blogs.nytimes.com/2011/12/26/the-old-order-changeth/.

Foucault, Michel. (2004). The Archeology of Knowledge. Trans. Tavistock Publications. London: Routledge Classics.

Gilroy, Paul. (1993). The Black Atlantic: Modernity and Double Consciousness. Cambridge: Harvard UP.

Gladney, Henry M. (2012). “Long-Term Digital Preservation: A Digital Humanities Topic?” Historical Social Research: Controversies around the Digital Humanities, 37.3: 201-17. http://www.jstor.org/stable/41636605.

Heuser, Ryan and Long Le-Khac. (2011). "Learning to Read Data: Bringing out the Humanistic in the Digital Humanities.” Victorian Studies, 54.1: 79-86. http://www.jstor.org/stable/10.2979/victorianstudies.54.1.79.

Jones, Robert Edmond. (2004). The Dramatic Imagination: Reflections and Speculations on the Art of Theatre. New York: Routledge.

Lei Zeng, Marcia. (2017). Smart Data for Digital Humanities. Vol. 2 No. 1. Pp. 1-12. DOI: 10.1515/jdis-2017-0001

Mahony, Simon. (2018). "Cultural Diversity and the Digital Humanities." Fudan J. Hum. Soc. Sci. doi.org/10.1007/s40647-018-0216-0.

Nelson, Theodor, H. (2003). “A File Structure for the Complex, the Changing, and the Indeterminate.” The New Media Reader. Ed. Noah Wardrip-Fruin and Nick Montfort. Cambridge: The MIT Press.

Presner, Todd. (2011). "Comparative Literature in the Age of Digital Humanities: On Possible Futures for a Discipline.” In A Companion to Comparative Literature. Ed. Ali Behdad and Dominic Thomas. Pp. 193-2017. Oxford: Blackwell Publishing.

Sabharwal, A. (2015). Digital Curation in the Digital Humanities. http://dx.doi.org/10.1016/B978-0-08-100143 $-1.00001-5$.

Simanowski, Roberto. (2016). 193-207. Digital Humanities and Digital Media: Conversations on Politics, Culture, Aesthetics and Literacy. London: Open Humanities Press.

Van Laar, Ester, Alexander J. A. M. van Deursen, Jan A. G. M. van Dijk, Jos de Haan. (2017). The relation between 21st-century skills and digital skills or literacy: A systematic literature review, The relation between 21st-century skills and digital skills or literacy A systematic literature review. doi: 10.1016/j.chb.2017.03.010.

Wells, H. G. (1938). A Short History of the World. Harmondsworth: Penguin. 